Conference abstract LPPT05

\title{
Pharmaceutical Hot Melt Extrusion
}

\author{
N. NeUB \\ Coperion $\mathrm{GmbH}$, Theodorstraße 10, 70469 Stuttgart, Germany \\ E-mail: nicole.neub@coperion.com \\ Sci Pharm. 2010; 78: 585 \\ doi:10.3797/scipharm.cespt.8.LPPT05
}

Hot Melt Extrusion, proven state-of-the-art technology in the field of plastics, is becoming increasingly important in the pharmaceutical industry. In the Hot Melt Extrusion process, the polymer is processed above the glass transition temperature to mix the active component with the thermoplastic binder and / or the polymer on a molecular level.

The most significant benefits of HME processing are the absence of water and solvents, which leads to fewer processing steps and lower manufacturing costs. In addition the short residence time is beneficial for many heat- and shear sensitive drugs and the free volume in the twin screw gives the processor the opportunity to devolatilise the compound in a controlled and reproducible environment.

Because of their excellent mixing behaviour and the degassing possibilities, corotating twin-screws are particularly suitable for Hot Melt Extrusion. The modular concept of the Twin Screw Extruder enables the individual adaptation of the processing section to the different requirements. Individual steps such as dispersive mixing, degassing and pressure build-up take place very effectively in this way.

The closely intermeshing screws with their tight, self-wiping profiles eliminate dead zones over the whole length of the process section. The effect: consistently higher degree of process reliability and optimal self-cleaning.

Coperion has references in the pharmaceutical field for twin screw extruders in sizes from 18 to $70 \mathrm{~mm}$ screw diameter.

Typical applications for Coperion's twin screw extruders ZSK:

- Hot Melt Extrusion

- Production of Transdermale Adhesives

- Extrusion of Micro-Pellets

- Production of Drugs with Delayed Ingredient Release

- Low-Temperature Melt Extrusion

- Potent-Extrusion

- Co-Extrusion 